\title{
Evolutionary prisoner's dilemma game with dynamic preferential selection
}

\author{
Zhi-Xi Wu ${ }^{1}$, Xin-Jian $\mathrm{Xu}^{2}$, Zi-Gang Huang ${ }^{1}$, Sheng-Jun Wang ${ }^{1}$, and Ying-Hai Wang ${ }^{1}$ \\ ${ }^{1}$ Institute of Theoretical Physics, Lanzhou University, Lanzhou Gansu 730000, China \\ ${ }^{2}$ Department of Electronic Engineering, City University of Hong Kong, Kowloon, Hong Kong SAR, China
}

(Dated: September 18, 2018)

\begin{abstract}
We study a modified prisoner's dilemma game taking place on two-dimensional disordered square lattices. The players are pure strategists and can either cooperate or defect with their immediate neighbors. In the generations each player update its strategy by following one of the neighboring strategies with a probability dependent on the payoff difference. The neighbor selection obeys a dynamic preferential rule, i.e., the more frequently a neighbor's strategy was adopted by the focal player in the previous rounds, the larger probability it will be chosen to refer to in the subsequent rounds. It is found that cooperation is substantially promoted due to this simple selection mechanism. Corresponding analysis is provided by the investigations of the distribution of players' impact weights, persistence, and as well as correlation function.

PACS numbers: 02.50.Le, 05.50.+q, 87.23.Cc, 89.65.-s
\end{abstract}

\section{INTRODUCTION}

Game theory and its evolutionary context are efficient frameworks to study complex behaviors of biological, ecological, social and economic systems [1, 2, 3, 4]. Of particular renown is the evolutionary prisoner's dilemma game (PDG) which has attracted much attention in theoretical and experimental studies [3, 4, 5, 6, 7]. In the original PDG, players can make two choices: either to cooperate with their co-players or to defect. They are offered some payoffs dependent on their choices, which can be expressed by a $2 \times 2$ payoff matrix in agreement with the four possibilities. The players get rewards $R$ (or punishment $P$ ) if both choose to cooperate (or defect). If one player cooperates while the other defects, then the cooperator $(C)$ gets the lowest payoff $S$ (sucker's payoff), while the defector $(D)$ gains the highest payoff $T$ (the temptation to defect). Thus the elements of the payoff matrix satisfy the conditions: $T>R>P>S$ and $2 R>T+S$, so that lead to a so-called dilemma situation where mutual cooperation is beneficial in a long perspective but egoism can produce big short-term profits.

One of the most interesting items on the PDG is to study under what conditions mutual cooperation will emerge and sustain stably or how to facilitate the cooperation of the whole population [2, 3, 4] . In the evolutionary PDG, the state that all players are defectors has been proved to be an evolutionary stable state [4], which has inspired numerous investigations of suitable extensions that enable cooperation to persist. Nowak and May [8] introduced a spatial evolutionary PDG model in which individuals located on a lattice play with their neighbors and with themselves. The dynamics is governed by a deterministic rule: in each subsequent round, individuals adopt the strategy that has gained the highest payoff among its neighbors (including also themselves) in the previous round. It has been shown that the spatial effects promote substantially the survival of cooperators $[8,9,10,11,12]$. The enhancement of cooperation on lattice-like spatial structure is robust even if some specified distance interactions are allowed [11].

Szabó and Tóke extended the deterministic dynamics to a stochastic evolutionary one [13]: rather than following the most successful neighbor's strategy straightly, the adoption of one of the neighboring strategies is allowed with a probability dependent on the payoff difference. This revised version took into account the irrational choices of the players. It was observed that a stable absorbing state of all $C$ emerged when the temptation to defect is below a certain critical value (noise-dependent). Vainstein and Arenzon studied the PDG on a diluted lattice [14] and found that cooperation is easier to maintain due to blocking the spreading of defection caused by the empty sites on the lattice. The investigations of the PDG on random graphs have shown that cooperation is strongly enhanced for lattices with fluctuating connectivity in comparison with fixed connectivity lattice [15]. In the past few years, the PDG has been studied on different social network models. It was found that cooperation can be maintained on these networks in a wide range of network parameters [16, 17, 18, 19, 20, 21, 22]. In addition, several mechanisms were also introduced to sustain high concentration of cooperators, such as voluntary participation [21], dynamic network model [23] and dynamic payoff matrices [24], and so on. Very recently, Santos et al. studied the PDG and snowdrift games on scale-free networks. Their results indicated that the heterogeneity of networks favors the emergence of cooperation [25].

Though many intriguing fruits have been obtained in understanding the emergence of cooperation in spatial PDG $[8,9,10,11,12,13,14,15,16,17,18,19,20,21,22]$, it would also be interesting to explore other mechanisms to enhance the cooperative behavior. In the present work, we make further studies of the evolutionary PDG using SzabóTőke version [13] on two-dimensional disordered square lattices. Considering individuals heterogeneously affected by their neighbors in society, we introduce impact weight to each one of the interacting players. Based on the player's impact weights, a dynamic preferential selection (DPS) mechanism is incorporated into the spatial PDG to model dynamic behaviors of human communities. It will be shown that the DPS promotes substantially the cooperative behavior of the players which may provide a new perspective in understanding the persistence of cooperation in realistic world.

In the next section, evolutionary rules of the game are explained. In Sec. III, simulation results of our model imple- 
mented on disordered lattices are presented and, in Sec. IV, the effects of the DPS on the evolution of cooperation is studied in detail. Conclusions are given in the last section.

\section{THE MODEL}

We consider the evolutionary PDG with players located on the two-dimensional disordered square lattice which is characterized by a total $\phi$ portion of randomly rewired links with fixed number of neighbors of each site (in the limit $\phi \rightarrow 1.0$, the "disordered lattice" is in fact a regular random graph [21, 26]). This approach excludes those effects coming from the fluctuation of the number of neighbors as it happens on diluted lattices [14], random graphs [15], and scale-free networks [25]. Similar consideration has also been made in Refs. [22, 26]. The players are pure strategists and can follow only two simple strategies: $C$ (cooperate) and $D$ (defect). Each player plays a PDG with itself and with its neighbors, and collects payoffs dependent on the payoff-matrix parameters. The total payoffs of a certain player is the sum over all interactions. Following common practices $[8,13,16,18]$, the elements of the payoff matrix can be rescaled, i.e., choosing $R=1, P=S=0$, and $T=b(1.0<b<2.0)$ to represent the advantage of defectors over cooperators. We have checked that the properties of the simulation results do not change for $S=-\epsilon(0.0<\epsilon \ll 1.0)$.

In society, some special persons may influence others much stronger than the average individual [18]. In other words, different neighbors would have different impacts on one's behavior. In general, one can expect that the influence between two individuals would be asymmetric and time-dependent. To model this situation, we define a quantity $A_{i j}(t)$, which describes the impact weight of the $j$ th player on the $i$ th player at time $t$ and possesses the asymmetric property, i.e., $A_{i j}(t) \neq$ $A_{j i}(t)$. In this way, we hope to catch some general effects of the dynamic asymmetric influence among interacting players on the dynamical behavior of the game.

After each round of the game, players are allowed to inspect their neighbors' payoffs and change their strategies in the next round according to the comparisons. The randomly chosen player $i$ revises its strategy by selecting one of its neighbors $j$ with a probability $\gamma$ in terms of a preferential selection rule:

$$
\gamma_{i j}=\frac{A_{i j}(t)}{\sum_{k \in \Omega_{i}} A_{i k}(t)},
$$

where $\Omega_{i}$ is the community composing of the nearest neighbors of $i$. Eq. (1) means that the stronger the impact of a neighbor is, the larger probability it is selected to compare with. If and only if their strategies are different, the $i$ th player's state as well as the neighbor's impact weight will be updated, otherwise nothing happens (no strategy transformation and impact weight update). Following ideas developed by Szabó et al. [13, 21, 22, 26], given the total payoffs ( $E_{i}$ and $E_{j}$ for the $i$ th and $j$ th players, respectively) from the previous round, the player $i$ adopts $j$ 's strategy with the probability

$$
W=\frac{1}{1+\exp \left[-\left(E_{j}-E_{i}\right) / \kappa\right]},
$$

where $\kappa$ characterizes the noise introduced to permit irrational choices. It should be noted that the transformation is only affected by their payoff difference. We set the value of $\kappa$ to 0.1 , similar to that in Refs. [13, 21, 22, 26]. Generating a random number $r$ from a uniform distribution in the interval $(0,1)$, if $r<W$ (or $r>W$ ), the player $j$ 's strategy is accepted (or discarded) by the player $i$ and $A_{i j}(t)$ is revised according to the following rule

$$
A_{i j}(t+1)=A_{i j}(t)(1 \pm \alpha),
$$

where the minus corresponds to the case of $r>W$, denoting no strategy updating for the $i$ th player. Initially, all $A_{i j}(0)$ are assigned to 1.0. The parameter $\alpha$ in Eq. (3) can be depicted as a multiplication factor which characterizes qualitatively the relative change of the impact weight in each round. Larger $\alpha$ corresponds to stronger strengthening (or losing) of impact. In subsequent investigations, the value of $\alpha$ is constrained in the range $(0,1)$ to ensure no negative elements in $A_{i j}(t)$, which means that all opinions ( $C$ and $D$ in the present case) have opportunities to be followed. If strategies of the players spread successfully, they can be called as "winners" regardless of the payoffs (for example, due to the noise, the worse performance strategy also has a certain probability to take over a better performance one). Thus, this rule (Eq. (3) could be termed as: "win-strengthen, lose-weaken". The more frequently a player's strategy followed by its neighbors, the larger probability it will be picked up to refer to in the subsequent generations, and vice versa.

\section{SIMULATION RESULTS}

In what follows three groups of systems will be considered: (i) regular square lattices $(\phi=0.0$ with $\alpha=0.0,0.01,0.1$, and 0.3 , respectively); (ii) weak disordered lattices ( $\phi=0.1$ with the same values of $\alpha$ as before); (iii) strong disordered lattices ( $\phi=1.0$ with the same values of $\alpha$ as the former). On the one hand (as a byproduct), we want to understand how the underlying disordered structures affect the evolution of the PDG; on the other hand (as the main aim), we try to explore the potentiality of the DPS (in combination with the disordered structures) on the evolution of cooperation.

All the simulations are performed in systems with $300 \times 300$ players. Starting from a random initial state with equal fraction of $C$ and $D$, we iterate the model with synchronous update. The evolution eventually leads to dynamic equilibrium states with small fluctuations of the cooperator density around an average value. The parameter $\alpha$, which determines the evolution of impact weights, is not critical, and convergence has been verified for all values of $\alpha$ between 0 and 1. A key quantity is the density of cooperators, $\rho_{C}$, which is defined as the average fraction of players adopting the strategy $C$ in the equilibrium state. The total sampling times were $10^{5}$ Monte-Carlo (MC) steps [27], and the equilibrium density of cooperators was obtained by averaging over the last $10^{4}$ steps. Each data point (in Figs. 11and 2) results from an average over 10 realizations for the same type of disordered lattices specified by the parameter $\phi$. 
The dependence of $\rho_{C}$ on $b$ in the equilibrium state for different values of $\phi$ and $\alpha$, are shown in Fig. 11. The main features in the steady state are similar to the results reported in Ref. [13], i.e., there exist two different absorbing states $\left(\rho_{C}=1\right.$ and $\left.\rho_{C}=0\right)$ separated by the active phase (coexistence of $C$ and $D$ ). We have found numerically that $\rho_{C}=1$ in all the cases we are interested in when $b<5 / 4$, which can be regarded as a homogeneous cooperation state. Since our main aim goes beyond this trivial steady-state, we will only concentrate on the region of $b>5 / 4$, where new features may emerge.

First, we consider the model without DPS ( $\alpha=0.0)$. In the case of $\phi=0.0$, which corresponds to the regular square lattice, we recover the results of the stochastic model [13], i.e. $\rho_{C}$ decreases monotonically with the increase of $b$ and vanishes at a threshold $b_{c}$. When long range links emerge on the lattice $(\phi=0.1)$, the level of cooperation is promoted (a larger threshold $b_{c}$ in Fig. 1(b) than that in Fig. 1(a)), which is different from previous reports that local interactions promote the sustainment of cooperation (see Refs. [8, 9, 10, 12] and the references therein). Particularly, in the case of $\phi=1.0$ (corresponding to a regular random graph), where the spatial correlation is very weak, cooperation is enhanced further (if it is just measured by the value of $b_{c}$ ). Even in the case of $b>2.0$, minor fractional cooperators can be found in a sea of defectors.

These results can be understood in the following way. On the one hand, in our model, the state transformation of the players conforms to a Fermi function (see Eq. (2) suggested by Szabó et al. [13, 21, 22, 26]. Using this update rule, they have also found that long range interactions enhance the cooperation when the PDG (with no self-interactions included) was performed on regular random graphs [21]. On the other hand, self-interactions are included in the present work. Recently, Hauert and Doebeli have studied another famous evolutionary game, snowdrift game, on different types of lattice [28]. They have found that the spatial structure eliminates cooperation for intermediate and high cost-to-benefit ratio of cooperation because benefits of costly cooperative acts accrue not only to others but also to the cooperator itself [28, 29]. As to our PDG model, each player $C$ plays with itself besides its nearest neighbors, which indicates that it will gain at least $R$ payoff even in the worst case (surrounding by four defectors). In a different interpretation, besides their neighbors, the cooperators' investment will benefit themselves too. Note that the high cost-to-benefit ratio of cooperation in snowdrift game corresponds to large values of $b$ in the PDG. Considering these two factors, it is not surprising that the disordered structure can promote cooperation in the present model.

We now focus our attention on the influence of the DPS on the game evolution. The results obtained for $\alpha=$ $0.01,0.1,0.3$ are summarized in Fig. 11. Although qualitative behaviors are similar to those of the random selection case, there are some remarkable quantitative differences. The simulation results depend strongly on both the parameters $\phi$ and $\alpha$. For well structured populations $(\phi=0$, regular square lattice case) with $\alpha=0.01$, the DPS promotes cooperation for small $b$ in comparison with the random selection case. For large $b$,

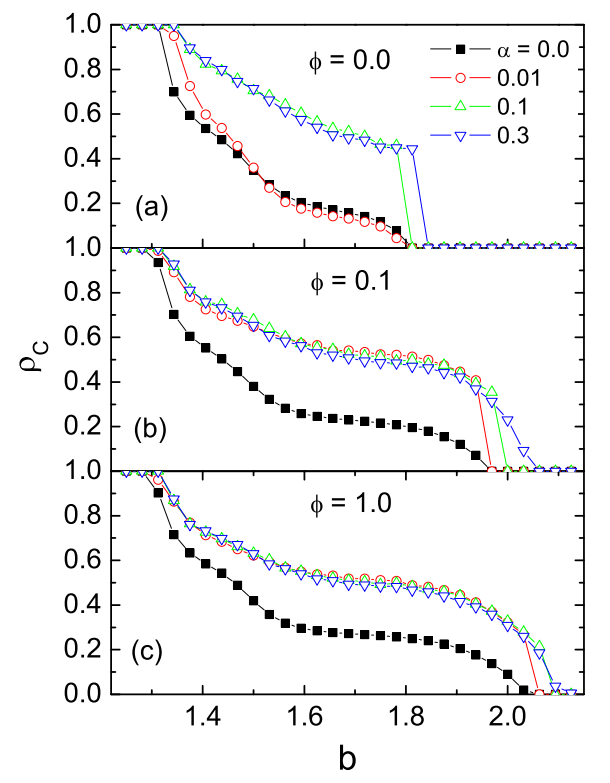

FIG. 1: (color online). Average density of cooperators, $\rho_{c}$, as a function of the temptation to defect $b$ in the equilibrium state. Filled and open symbols correspond to the cases of random selection and preferential selection, respectively.

however, the tendency is reversed and the fraction of cooperators is somewhat lower than the corresponding value in the case of $\alpha=0.0$. There exists a cross point for $b$ separating the two regions which depends on $\alpha$ : for $\alpha=0.01, b \approx 1.5$. For the other two values of $\alpha(0.1$ and 0.3$)$, the DPS enhances greatly the cooperation with respect to otherwise. In the cases of disordered structural populations ( $\phi=0.1$ and 1.0), for all the values of $\alpha$ considered here, the cooperative behavior can be substantially promoted (see Figs. 1 b) and 1 (c)) and maintained even in the extreme defection circumstance $(b>2.0)$, where the game is not a proper Prisoner's Dilemma. It is strikingly interesting that, for disordered structural populations, all the curves nearly collapse into a single curve (shown in Figs. 1 (b) and 1 (c)) when the DPS is introduced into the game. The visible difference exists only in the region where cooperators are going to extinction. The larger $\alpha$ gives rise to larger threshold $b_{c}$. The data collapsing is also found in well structured populations for large $\alpha$ (see Fig. 1 (a)).

From these observed features, we argue that, whether the underlying "lattice"is well structured or disordered, as soon as the multiplication factor $\alpha$ is finite and sufficiently large, the enhancement of cooperation will be to some extent realized. Despite of the fact that the disordered structure prefers to favor the emergence of cooperation, the much stronger enhancement of cooperative behavior for $\alpha>0.0$ than that for $\alpha=0.0$ implies that the DPS plays a crucial role in the dynamics.

In Fig. 2, we show the plots of $\rho_{C}$ as a function of $\alpha$ for three special values of $b$, to emphasize the changes in behaviors as the lattice varies. The colored dashed lines correspond 


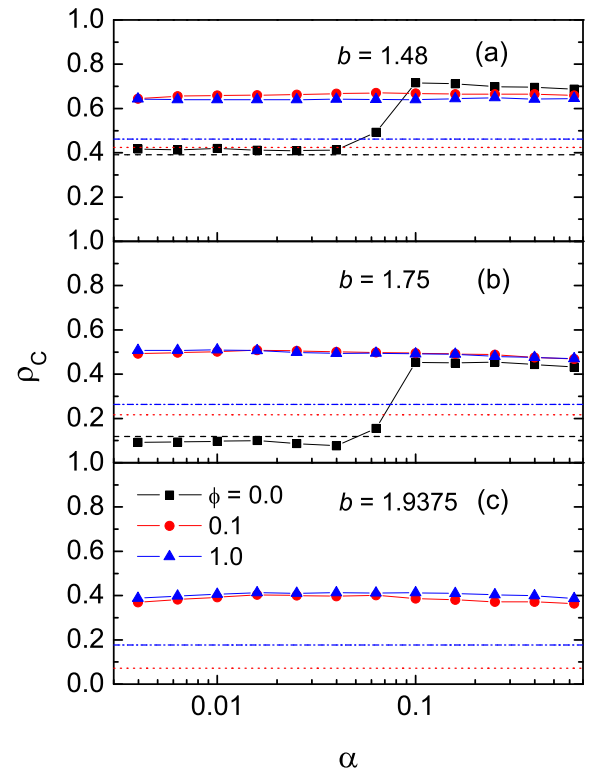

FIG. 2: (color online). Average density of cooperators, $\rho_{c}$, as a function of the multiplication factor, $\alpha$, for three special values of $b$ (colored symbols). The colored lines correspond to random selection cases $(\alpha=0.0)$ : dashed (black) for $\phi=0.0$, dotted (red) for $\phi=0.1$, and dash-dotted (blue) for $\phi=1.0$, respectively.

to the results obtained for the random selection case and the symbols for the DPS case. Different colors denote different disordered degree of the lattice: black for $\phi=0.0$, red for $\phi=0.1$ and blue for $\phi=1.0$, respectively. Note that only the curves for $\phi=0.0$ have a clear sensitivity to the values of $\alpha$ (black squares in Figs. 22a) and 2 (b)). The temptation to defect, $b$, has also an influence on the results. In the cases of small $\alpha$ with $\phi=0.0$, for low temptation $(b=1.48)$, the cooperation level is slightly higher for the DPS case than that for the random selection case; whereas for high temptation $(b=1.75)$, the phenomenon is reversed. The Similar discrepancy can also be found for large values of $\alpha$ with $\phi=0.0$ : the spatial structure promotes slightly the cooperation level for $b=1.48$ (see Fig. 2a)), and yet inhibits that for $b=1.75$ (see Fig. 2(b)). The transition point seems to be around $\alpha \approx 0.06$. Since regular lattices are not realistic representations of most actual population structures [30], especially human (because of the mobility and dispersal ability of the individuals), we do not comment further on these trivial results and focus our attention on those issues where disordered structures are present $(\phi=0.1$ and 1.0).

Now taking a look at Figs. 2(a), (b) and (c), we clearly observe that for any finite values of $\alpha$ we are interested in, given fixed values of $b$ and $\phi$, the cooperation level is almost independent of $\alpha$, and the relative deviation of the results is less than $5 \%$. Despite of this point, we would like to point out that the enhancement of cooperation appears also insensitive to the special values of $\phi$, i.e., more disordered structures have little essential influence on the evolutionary results, at least,
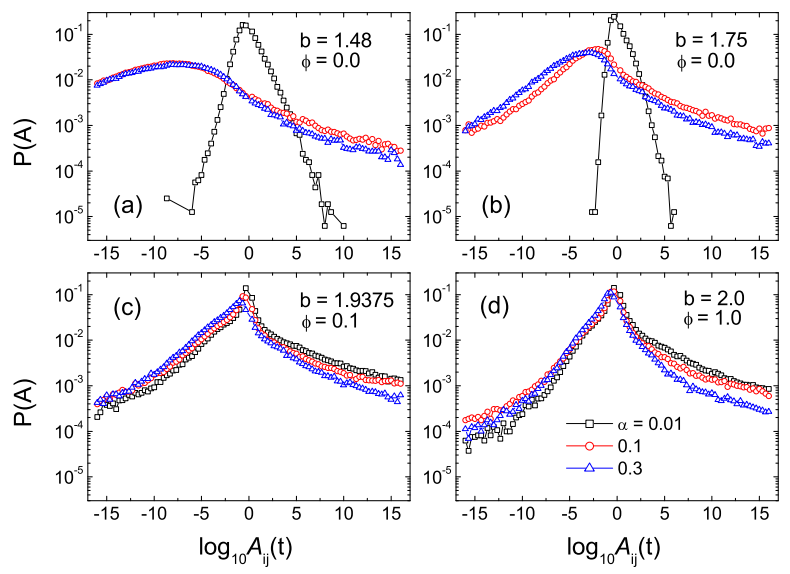

FIG. 3: (color online). Histograms (over $10^{5}$ time steps) of impact weights of players in the equilibrium state. Only the data which fall into the region $\left[10^{-16}, 10^{16}\right]$ are shown. The power-law decaying behavior determined by the intrinsic nature of Eq. (3) is visible in both the very large limit and the very small limit of the impact weights.

for the case of $0.1<\phi<1.0$. This problem should deserve to make further investigations in the future work.

To end up this section, let us review the novel features of the results obtained by computer simulations. When introducing the DPS to the evolutionary PDG, we have found that the cooperative behavior in the populations is substantially enhanced with respect to the random selection case. The enhancement is robust against whether the underlying lattice is well structured or disordered. Though the evolutionary results depend on both $\phi$ and $\alpha$, as long as the underlying lattice is finitely disordered and the multiplication factor $\alpha$ is also sufficiently large (e.g., $\alpha>0.003$ in the present studied cases [31]), the resulting cooperation level seems insensitive to both $\phi$ and $\alpha$ for fixed values of $b$.

\section{ANALYSIS AND DISCUSSION}

Before discussing the further details as to why the above evolutionary results may occur, we briefly review those results obtained for the evolutionary PDG performed on the regular lattice. Generally, we are more interested in the region near the extinction threshold of cooperators, from which we can figure out clearly cooperators' evolution and surviving in the sea of defectors. For this issue, Hauert et al. [21, 28] have found that cooperators can survive by forming large, compact clusters which minimize the exploitation by defectors. Along the boundary, cooperators can outweigh their losses against defectors by gains from interactions within the cluster . Similar phenomena were observed earlier by Szabó and Tóke [13]. The compact clusters composing of cooperators do random walks and spread out in the background of defectors. Though, occasionally a cluster splits into two or two clusters collide, merge, or annihilate and vanish [21], the compact structures 

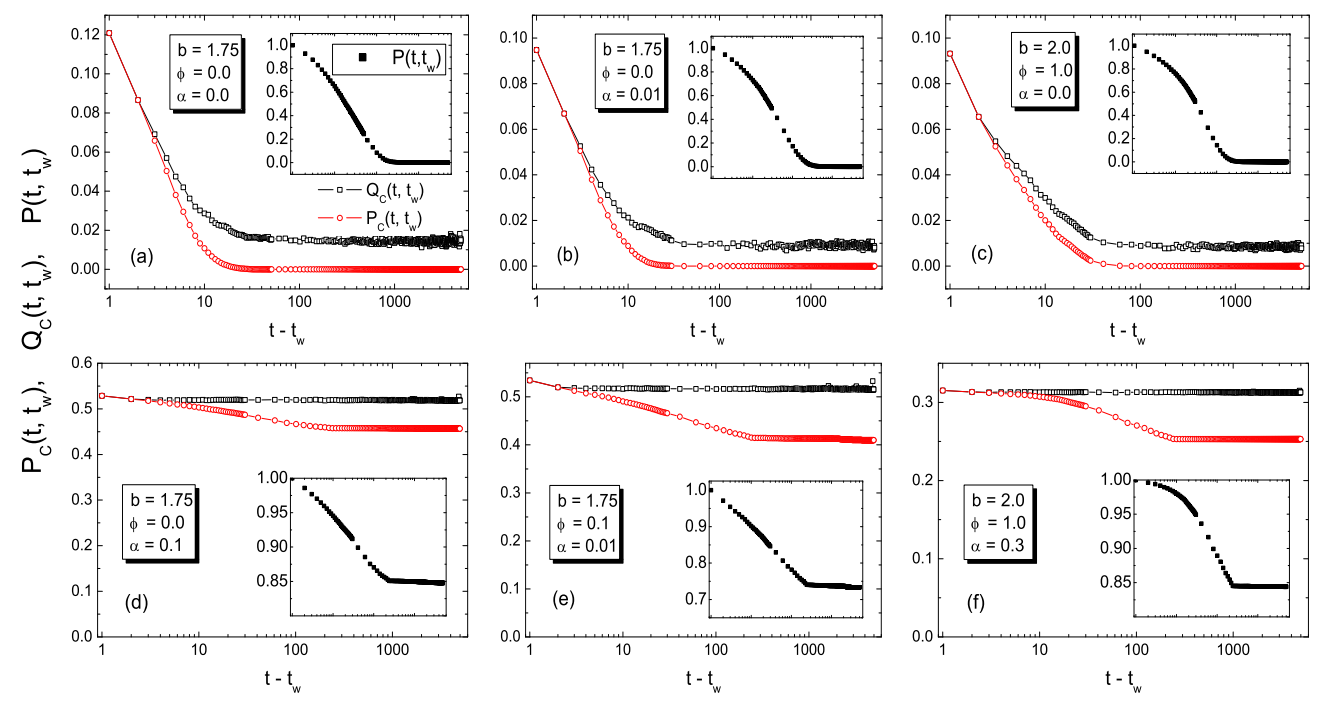

FIG. 4: (color online). Persistence function $P_{c}\left(t, t_{w}\right)$ and correlation function $Q_{c}\left(t, t_{w}\right)$ for different combinations of $b, \phi$ and $\alpha$ as a function of time interval $t-t_{w}$ where $t_{w}=95000$. Inset: time evolution of the persistence function $P\left(t, t_{w}\right)$ for both strategies $C$ and $D$. That the persistence $P_{c}\left(t, t_{w}\right)$ goes to zero in the long time limit indicates the random walk and annihilation of cooperators, whereas the non-zero plateau of it implies the stable maintenance of the communities of the cooperators. The plots of $Q_{c}\left(t, t_{w}\right)$ also give the same hints, which sustains the level of the datum time $t_{w}$ for the stable maintenance case, and displays the mean-field behavior for the random walk case. See the text for details.

of the surviving clusters can remain in principle unchanged.

The problem can be viewed more explicitly: when the players' strategy update is implemented by randomly selecting a neighbor to compare with according to Eq. (3), the compact structures favor those internal cooperators to forming a stable core, since the components interact only with players taking over strategy $C$. The existence of this core enables those cooperators on surface to have enough channels to collect payoffs so that they can resist the invading of defectors, which in return reinforce the stability of the core. The mutual protection (or reciprocity) of the cooperators enables the compact structures to sustain stably, and hence contributes to the persistence of cooperation.

Motivated by the above interpretations, some conjecture on the origin of those results of our model with the presence of DPS depicted in Sec. III may be appropriate here. We think that the DPS could induce the emergence of pairs of influential co-players, and when some of them are cooperators, they will "always"refer to their influential neighbors' strategies in the subsequent generations, then communities consisting of their neighbors and themselves may form and survive stably in the background of defectors, which would contribute to the persistence of cooperation. Those influential co-players taking over strategy $C$ can be regarded as the core of the communities (or clusters). To test this speculation, we check the probability distribution of $A_{i j}(t)$ in the equilibrium state, which is expected to have a broad distribution so that there could arise a large enough fraction of influential co-players (being cooperators) to form stable core in populations.

In Figure 3, we plot probability distributions of $A_{i j}(t)$ for four different combinations of $b$ and $\phi$ and with corresponding three values of $\alpha: b=1.48, \phi=0.0$ (Fig. 3(a)); $b=1.75$, $\phi=0.0$ (Fig. 3 b)); $b=1.9375, \phi=0.1$ (Fig. 3(c)); and $b=2.0, \phi=1.0$ (Fig. 3 d)), respectively. As indicated, there are very large broad distributions of $A_{i j}(t)$ except for two combinations, $(b=1.48, \phi=0.0, \alpha=0.01)$ and $(b=1.75, \phi=0.0, \alpha=0.01)$. These two combinations do not favor the emergence of cooperation (see Fig. 11 a) for the symbols of red circles) in contrast to other combinations, and also do not provide sufficient conditions for players to form stable clusters which can be seen in the following part. It is worthy to point out that the combinations of well structured populations with sufficiently large $\alpha$ values (Figs. 3 a) and 3 b)) or disordered populations with finite $\alpha$ values (Figs. 3 (c) and 3(d)) resulting in broad histograms of $A_{i j}(t)$ indeed facilitate the cooperative behavior compared with corresponding random selection cases $(\alpha=0.0)$, which have been verified by previous simulations (see Figs. 11and2).

To prove that some fractional players may form stable clusters in the presence of the DPS in a more detailed way, we measured the persistence function $P_{C}\left(t, t_{w}\right)$, the fraction of cooperators that do not change strategy between an initial waiting time $t_{w}$, and the time $t \geq t_{w}$ [14]. In addition, the correlation function $Q_{C}\left(t, t_{w}\right)$, which characterizes the frac- 

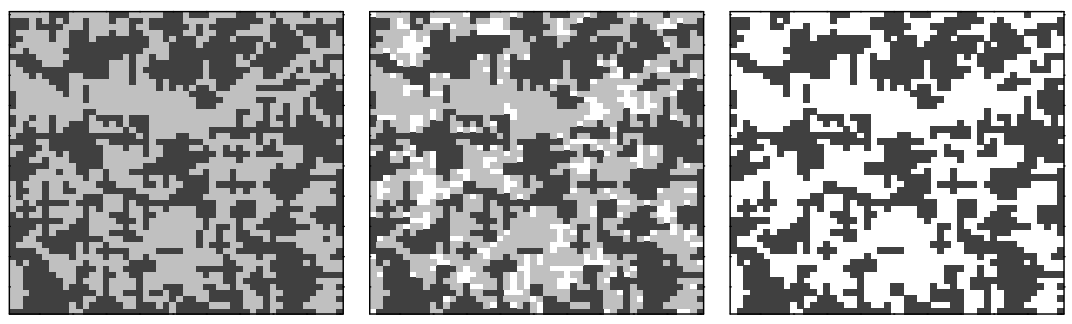

FIG. 5: Right panel: illustration of cooperators and defectors at time $t=9.5 \times 10^{4}$. Middle panel: snapshot showing players that remain unchanged between MC time $t=9.5 \times 10^{4}$ and $10^{5}$ : the pinned cooperators, defectors, and the active players are denoted by dark gray boxes, light gray boxes, and white vacancy, respectively. Right panel: only the pinned cooperators are shown. Parameters: $\phi=0.0, b=1.75, \alpha=$ 0.1 .

tion of cooperators at time $t$ that have arisen at time $t_{w}$ in spite of finitely many times flipping of the strategy during the two time interval, is also explored. In a distinct view, the results of these two quantities as a function of the time interval $t-t_{w}$ (measured in MC steps, the time $t_{w}$ can be selected arbitrarily as long as the systems had attained equilibrium) are summarized in Fig. 4 on a log-linear scale for $t_{w}=95000$.

For the cases of well structured populations with sufficiently large $\alpha$ (Fig. 4(d)) or disordered populations with finite $\alpha$ (Figs. 4(e) and 4(f)), after an initial decrease, the persistence attains, for large time intervals, a plateau whose value depends on the parameters $b, \phi$ and $\gamma$. Interestingly, for a nonHamiltonian model we studied here, the decaying behavior of the persistence in the equilibrium is exponential at large time intervals, just like that has been observed commonly in Hamiltonian models and in site diluted lattice model [32]. If the persistence does not go to zero, there is a fraction of sites of cooperators that flip only finitely many times (blocking), and domain wall movements are constrained (pinning) [14]. For the present systems we studied, it indicates that communities of cooperators exist stably in the background of defectors. In the cases of random selection (no matter whether the populations are well structured or disordered, Figs. 4(a) and 4(c)) or well structured populations with very small $\alpha$ (Fig. 4(b)), however, the persistence goes to zero in the long time interval limit, which means that all the cooperators are renewed completely after finite waiting time. This is reminiscent of the random walk and annihilation [13]. The persistence function $P\left(t, t_{w}\right)$ for both strategies $C$ and $D$ shown in insets also displays the similar characteristics.

The time dependence of the correlation function $Q_{C}\left(t, t_{w}\right)$ also reflects the same evolving behavior of the systems. For well structured populations with sufficiently large $\alpha$ (Fig. $4(d))$ or disordered populations with finite $\alpha$ (Figs. 4(e) and $4(\mathrm{f})), Q_{C}\left(t, t_{w}\right)$ fluctuates weakly around the average fraction of cooperators, $\rho_{C}$, indicating the stable maintenance of the communities of cooperators. For the random selection case (Figs. 4a) and 4(c)) or well structured populations with very small $\alpha$ (Fig. 4(b)), the random walks and annihilation of cooperators causes the long time correlation to be independent of the initial state, which can be calculated roughly by a mean-field method. Since the spread of cooperators can be regarded as walking randomly in such cases, the probability of revisiting those sites which had been visited before will be proportional to the average density of cooperators. Assuming that the fraction of cooperators is equal to $\rho\left(t_{w}\right)$ at time $t_{w}$ (the system has already attained equilibrium before this time), the mean-field approximation gives the relation, $Q_{C}\left(t, t_{w}\right) \approx \rho_{C} \times \rho\left(t_{w}\right)$, in the long time limit, $t \rightarrow \infty$. As shown in Figs. 4(a), 4(b) and 4(c), the analysis is in well agreement with numerical simulations.

For an intuitive understanding of the surviving of cooperators, in Fig. 5 we present snapshots of the system in the equilibrium state under the condition of $\phi=0, b=1.75, \alpha=0.1$, showing both active and pinned players. These snapshots are a $50 \times 50$ portion of the full $300 \times 300$ lattice. Cooperators and defectors are shown in dark gray and light gray boxes, respectively. The white vacancy denotes active players. This typical configuration is almost stable and the small amount of active sites is confined to a few regions. It is shown that most cooperator clusters remain unchanged confirming previous analysis.

At last, it should be pointed out that it is only qualitatively depicted that the large broad distributions of the impact weights favor the formation of stable clusters. The quantitative analysis on this issue, however, is unimplemented. The work along this line is in progress.

\section{CONCLUSIONS}

In this work, we have explored the general question of cooperation formation and sustainment from the perspective of the coevolution between the dynamics of players' states and their inter-influential relationships. By considering asymmetric and heterogenous influential effects in many natural populations, we defined impact weights for any pairs of neighbor- 
ing individuals, which describes the influence of one player on another and evolves timely. Based on this quantity, a dynamic preferential selection (DPS) mechanism was introduced to the dynamics of an evolutionary PDG. To simulate the mobility and dispersal ability of individuals, the disordered structure of populations was also taken into account.

Although disordered structure has been proved to favor the emergence of cooperation, it was found that the DPS plays a crucial role in determining the evolutionary results of the game. In fact, for well structured populations with strong multiplication effects (large $\alpha$ ) or disordered structural populations with the presence of the DPS, the cooperative behavior can be substantially promoted. These findings are presented for some specific sets of parameter values of $b, \phi$ and $\alpha$, but they are qualitatively the same for a broad range of values. By analyzing the probability distribution of $A_{i j}(t)$ in the long time limit, the persistence function $P_{C}\left(t, t_{w}\right)$ and the correlation function $Q_{C}\left(t, t_{w}\right)$ for the cooperators in the equilibrium state, we found that the DPS gives rise to very large broad distributions of the impact weights, which favor the influential cooperators to form stable communities, and thereby con- tribute to the emergence and persistence of cooperation. One interesting result is that the enhancement of cooperation, once realized, is insensitive to some special values of $\phi$ and $\alpha$. That is to say, the enhancement of cooperation in the presence of the DPS is general and robust, especially for finitely disordered structural populations. Another intriguing result is that, given the appropriate multiplication factor $\alpha$, cooperators can always persist in the whole range of $1.0<b<2.0$ for disordered structural populations, providing an escape hatch out of states of mutual defection predicted by both classical and evolutionary game theory. Thus, the DPS is capable of changing the doomed fate of cooperators and ensuring them to persist even under harsh conditions when $b \rightarrow 2.0$. Although it is simple, the present model offers an efficient way to simulate real social behaviors, and might shed lights in understanding the evolution of cooperation in complex social systems where asymmetry, heterogeneity, and feedback are ubiquitous.

This work was supported by the Fundamental Research Fund for Physics and Mathematics of Lanzhou University under Grant No. Lzu05008.
[1] J.von Neumann and O. Morgenstern, Theory of Games and Economic Behavior (Princeton University Press, Princeton, NJ, 1953).

[2] J.M. Smith, Evolution and the Theory of Games (Cambridge University Press, Cambridge, 1982).

[3] R. Axelrod, The Evolution of Cooperation (Basic Books, New York, 1984).

[4] J. Hofbauer and K. Sigmund, Evolutionary Games and Population Dynamics(Cambridge University Press, Cambridge, 1998).

[5] L.M. Wahl and M.A. Nowak, J. Theor. Biol. 200, 307 (1999).

[6] E. Fehr and U. Fischbacher, Econom. J. 112, 478 (2002).

[7] M. Mesterton-Gibbons and L.A. Dugatkin, Anim. Behav. 54, 551 (1997).

[8] M.A. Nowak and R.M. May, Nature (London) 359, 826 (1992); Int. J. Bifurcation Chaos Appl. Sci. Eng. 3, 35 (1993).

[9] B.A. Hubermann and N.S. Glance, Proc. Natl. Acad. Sci. U.S.A. 90, 7712 (1993).

[10] M.A. Nowak, S. Bonhoeffer, and R.M. May, Proc. Natl. Acad. Sci. U.S.A. 91, 4877 (1994).

[11] M.A. Nowak S. Bonhoeffer, and R.M. May, Nature (London) 379, 125 (1996).

[12] C. Hauert, S.D. Monte, J.Hofbauer, and K. Sigmund, J. theor. Biol. 218, 187 (2002).

[13] G. Szabó and C. Töke, Phys. Rev. E 58, 69 (1998).

[14] M.H. Vainstein and J.J. Arenzon, Phys. Rev. E 64, 051905 (2001).

[15] O. Duran and R. Mulet, e-print cond-mat/0305353

[16] G. Abramson and M. Kuperman, Phys. Rev. E 63, 030901(R) (2001).

[17] H. Ebel and S. Bornholdt, Phys. Rev. E 66, 056118 (2002).

[18] B.J. Kim, A. Trusina, P. Holme, P. Minnhagen, J.S. Chung, and M.Y. Choi, Phys. Rev. E 66, 021907 (2002).

[19] P. Holme, A. Trusina, B. J. Kim, and P. Minnhagen, Phys. Rev.
E 68, 030901(R) (2003).

[20] Z.X. Wu, X.J. Xu, Y. Chen, and Y.H. Wang, Phys. Rev. E 71, 037103 (2005).

[21] C. Hauert and G. Szabó, Am. J. Phys. 73, 405 (2005); G. Szabó and C. Hauert, Phys. Rev. Lett. 89, 118101 (2002); Phys. Rev. E 66, 062903 (2002).

[22] G. Szabó and J. Vukov, Phys. Rev. E 69, 036107 (2004); J. Vukov and G. Szabó, Phys. Rev. E 71, 036133 (2005).

[23] M.G. Zimmermann, V.M. Eguiluz, and M. SanMiguel, Phys. Rev. E 69, 065102(R) (2004); M.G. Zimmermann and V.M. Eguiluz, Phys. Rev. E 72, 056118 (2005); H. Ebel and S. Bornholdt, e-print cond-mat/0211666.

[24] M. Tomochi and M. Kono, Phys. Rev. E 65026112 (2002).

[25] F.C. Santos and J.M. Pacheco, Phys. Rev. Lett. 95098104 (2005); F.C. Santos, J.M. Pacheco, and Tom Lenaerts, Proc. Natl. Acad. Sci. U.S.A. 103, 3490 (2006).

[26] G. Szabó, J. Vukov, and A. Szolnoki, Phys. Rev. E 72 047107 (2005); J. Vukov, G. Szabó, and A. Szolnoki, e-print cond-mat/0603419

[27] For $\alpha>0.003,10^{5} \mathrm{MC}$ steps are sufficient for the systems to attain equilibrium. For smaller $\alpha$, a much longer relaxation time is needed.

[28] C. Hauert and M. Doebeli, Nature (London) 428, 643 (2004).

[29] M. Doebeli, C. Hauert, and T. Killingback, Science 306, 859 (2004).

[30] M.E.J. Newman, SIAM Review 45, 167 (2003).

[31] We have also simulated the PDG for the cases of $\alpha<0.003$. The enhancement of cooperation and convergence have also been verified. However, the MC time needed for the systems to attain equilibrium increases dramatically.

[32] See, for example, Ref. [14] and references therein. 